\title{
Property Improvement of a-Amylase from Bacillus stearothermophilus by Deletion of Amino Acid Residues Arginine 179 and Glycine 180
}

\section{Yuanming Gai', Jingqi Chen ${ }^{1,2}$, Shibin Zhang ${ }^{1,2}$, Beiwei Zhu ${ }^{3}$ and \\ Dawei Zhang 1,2,3*}

${ }^{1}$ Tianjin Institute of Industrial Biotechnology, Chinese Academy of Sciences, 32 West 7th Avenue, Tianjin Airport Economic Area, 300308 Tianjin, PR China

${ }^{2}$ Key Laboratory of Systems Microbial Biotechnology, Chinese Academy of Sciences, 32 West 7th Avenue, Tianjin Airport Economic Area, 300308 Tianjin, PR China

${ }^{3}$ School of Food Science and Technology, Dalian Polytechnic University, National Engineering Research Center of Seafood, No. 1st Qinggongyuan, Ganjingzi, 116034 Dalian, PR China

Received: July 30, 2017 Accepted: January 22, 2018

\section{*Corresponding author: \\ Phone: +862224828749; \\ Fax: +862224828749; \\ E-mail:zhang_dw@ tib.cas.cn}

ORCID IDs: 0000-0002-3234-5914 (Gai), 0000-0003-0750-8539 (Chen), 0000-0001-7935-5637 (Zhang, S), 0000-0003-4674-2993 (Zhu), 0000-0002-5857-

$-394 X$ (Zhang, D)

Paper was presented at the 7th International Forum on Industrial Bioprocessing - IFIBiop 2017, May 21-24, 2017, Wuxi, PR China

\section{SUMMARY}

To improve the properties of a-amylase from Bacillus stearothermophilus (AmyS), a deletion mutant AmySAR179-G180 was constructed by deleting arginine (Arg179) and glycine (Gly180) using site-directed mutagenesis. AmyS and AmyS $\triangle R 179-G 180$ were expressed in Bacillus subtilis and purified by ammonium sulfate precipitation, after which the enzymatic properties were characterized and compared. By deleting amino acids Arg179 and Gly180, the thermostability of $a$-amylase AmyS $\triangle R 179-G 180$ was enhanced and the half-life at 100 ${ }^{\circ} \mathrm{C}$ significantly increased from 24 to $33 \mathrm{~min}$. In addition, AmyS $\triangle \mathrm{R} 179-\mathrm{G} 180$ exhibited greater acid resistance and lower calcium requirements to maintain a-amylase activity. The secretory capacity of the recombinant strain was evaluated by fed-batch fermentation in a 7.5-litre fermentor in which high a-amylase activity was obtained. The highest activity reached $3300 \mathrm{U} / \mathrm{mL}$ with a high productivity of $45.8 \mathrm{U} /(\mathrm{mL} \cdot \mathrm{h})$.

Key words: a-amylase, Bacillus subtilis, fermentation, site-directed mutagenesis, thermostability

\section{INTRODUCTION}

a-Amylases (1,4-a-D-glucan glucanohydrolases; EC 3.2.1.1) are classical calcium-binding enzymes, which randomly hydrolyze internal a-1,4-glucosidic linkages in starch to produce smaller molecular mass maltodextrins, maltooligosaccharides and glucose (1). a-Amylases have great commercial value, as they can be used in the initial stages of starch processing (liquefaction) in the production of fructose and glucose syrups, detergent matrices as cleaning agents, in the baking industry for improving flour and in the textile industry for the removal of starch from textiles (2).

In the industrial production of glucose and fructose, starch liquefaction by a-amylases and saccharification by $\beta$-amylases are carried out sequentially. During liquefaction, the $\mathrm{pH}$ must be adjusted to between 5.5 and 6.0 and calcium ions need to be added due to the poor properties of widely used a-amylases produced by wild type strains such as Bacillus amyloliquefaciens (BAA) (3), Bacillus stearothermophilus (BSA) (4) and Bacillus licheniformis (BLA) (5), which significantly increase the production cost of glucose and fructose. Hence, much work has been performed on protein engineering, including random mutagenesis, site-directed mutagenesis, and DNA shuffling, all of which have been widely applied to improve thermostability, reduce operating $\mathrm{pH}$ range and remove the calcium requirement of a-amylases to meet the needs of industrial production (6). While random mutagenesis and DNA shuffling require highly efficient screening methods, rational design, based on sequence alignment and structural analysis of site-directed mutagenesis, is an effective method to obtain beneficial mutants. In previous studies, the thermostability of BAA (7) and Bacillus KSM-1378 a-amylase (LAMY) (8) was greatly improved by deletion of an Arg-Gly peptide. Furthermore, a truncated Bacillus sp. strain TS-23 a-amylase (BAC $\triangle N C)$ (9) had the same effect by deleting Arg210-Ser211. The half-life of $B$. stearothermophilus AmyUS100 was also increased by the deletion of lle214-G215 (10).

In this study, we deleted the amino acid residues Arg179 and Gly180 in AmyS by site-directed mutagenesis to optimize the properties of AmyS. The mutant AmyS was found to be 
more acid-resistant at $\mathrm{pH}=4.5-6.0$ than the wild type AmyS, the thermostability was significantly improved and the requirement for calcium to maintain activity was decreased. Furthermore, we used a 7.5-litre fermentor to evaluate the recombinant production of AmyS $\triangle \mathrm{R} 179-\mathrm{G} 180$ in fed-batch cultivation.

\section{MATERIALS AND METHODS}

\section{Materials}

Peptone and yeast extract were obtained from Oxoid, Thermo Fisher Scientific (Basingstoke, UK). Coomassie Brilliant Blue R-250 staining solution was obtained from Bio-Rad (Hercules, CA, USA). The 3,5-dinitrosalicylic acid (DNS) was obtained from Sigma-Aldrich (St Louis, MO, USA). $\mathrm{NaCl}$, $\mathrm{CaCl}_{2^{\prime}} \mathrm{K}_{2} \mathrm{HPO}_{4^{\prime}} \mathrm{KH}_{2} \mathrm{PO}_{4^{\prime}}\left(\mathrm{NH}_{4}\right)_{2} \mathrm{SO}_{4^{\prime}} \mathrm{MgSO}_{4} \cdot 7 \mathrm{H}_{2} \mathrm{O}$, sodium acetate, sodium citrate, sodium phosphate, starch, glucose, L-tryptophan, acid-hydrolyzed casein and antibiotics were purchased from Beijing Solarbio Science \& Technology Co., Ltd. (Beijing, PR China). NuPAGE $12 \%$ Bis-Tris Gel and MOPS SDS Running Buffer were obtained from Invitrogen (Carlsbad, CA, USA). PrimerSTAR Max DNA Polymerase and Dpnl were purchased from Takara Biotechnology (Dalian) Co., Ltd. (Dalian, PR China).

\section{Plasmids, strains, medium and culture conditions}

Plasmids and bacterial strains used in this study are described in Table 1. The pMA5 is an Escherichia coli/B. subtilis shuttle plasmid, which was donated by the Bacillus Genetic Stock Center (Columbus, OH, USA). E. coli DH5a was chosen as a host for molecular cloning and plasmid construction. This strain was cultivated in Luria-Bertani (LB) medium at $37^{\circ} \mathrm{C}$, which contained $5 \mathrm{~g} / \mathrm{L}$ of yeast extract, $10 \mathrm{~g} / \mathrm{L}$ of peptone and $5 \mathrm{~g} / \mathrm{L}$ of $\mathrm{NaCl}$. B. subtilis $1 \mathrm{~A} 751$ was used as the host strain for AmyS (a-amylase from B. stearothermophilus) expression, and this strain was grown in Super rich (SR) medium (2.5\% yeast extract, $1.5 \%$ peptone and $0.3 \% \mathrm{~K}_{2} \mathrm{HPO}_{4^{\prime}} \mathrm{pH}=7.2$ ) at $37^{\circ} \mathrm{C}$. Antibiotics were added as follows: kanamycin $25 \mu \mathrm{g} / \mathrm{mL}$ and ampicillin $100 \mu \mathrm{g} / \mathrm{mL}$.
The components of the medium used for Bacillus subtilis transformation were as follows: Spizizen minimal medium consisting of (in g/L): $\left(\mathrm{NH}_{4}\right)_{2} \mathrm{SO}_{4} 2, \mathrm{KH}_{2} \mathrm{PO}_{4} 6, \mathrm{~K}_{2} \mathrm{HPO}_{4} 18.3$ and sodium citrate 1.2. GMI (growth medium I) was Spizizen minimal medium supplemented with $0.8 \%$ glucose, $0.04 \%$ acid-hydrolyzed casein, $0.1 \%$ yeast extract, $0.02 \% \mathrm{MgSO}_{4} \cdot 7 \mathrm{H}_{2} \mathrm{O}$ and $0.005 \%$ L-tryptophan, and GMII (growth medium II) was Spizizen minimal medium supplemented with $0.8 \%$ glucose, $0.02 \%$ acid-hydrolyzed casein and $0.16 \% \mathrm{MgSO}_{4} \cdot 7 \mathrm{H}_{2} \mathrm{O}$.

\section{Site-directed mutagenesis}

The Easy Mutagenesis System (TransGen Biotech Co. Ltd., Beijing, PR China) was used to construct the mutant plasmid in accordance with the supplier's instructions. First, a linear plasmid carrying the desired mutation was PCR-amplified with primers AmyS $\triangle$ R179-G180-F (5'-TTACATATGAAACAACAAAAACGGCTTTACG-3') and AmySAR179-G180-R (5'-CGCGGATCCTTAAGGCCATGCCACCAGTC-3') with PrimerSTAR Max DNA Polymerase using the plasmid pMA5-AmyS as a template. The PCR products were then digested with Dpnl, which degraded the methylated DNA, removing the template. Finally, the nicked plasmid DNA carrying the desired mutation was transformed into E. coli DH5a, according to the methods described by Green and Sambrook (11). The newly constructed plasmid was designated pMA5-AmyS RR179-G180 and verified by DNA sequencing (12) (Genewiz, Suzhou, PR China).

\section{B. subtilis transformation}

First, GMI and GMII were prepared as mentioned previously (13). B. subtilis $1 \mathrm{~A} 751$ and derivatives were cultivated in GMI overnight at $37^{\circ} \mathrm{C}$. A volume of $0.5 \mathrm{~mL}$ of overnight culture was transferred into $4.5 \mathrm{~mL}$ of GMI medium and grown at 37 ${ }^{\circ} \mathrm{C}$ for $4.5 \mathrm{~h}$. A volume of $0.75 \mathrm{~mL}$ of culture was then mixed with $4.25 \mathrm{~mL}$ of GMII medium and the culture was incubated for $1.5 \mathrm{~h}$ at $37^{\circ} \mathrm{C}$. A mass of plasmid of $1 \mu \mathrm{g}$ was mixed with 1 $\mathrm{mL}$ of competent cells and incubated for $1 \mathrm{~h}$ at $37^{\circ} \mathrm{C}$. The cells were then plated on LB agar plates containing kanamycin $(25$ $\mu \mathrm{g} / \mathrm{mL}$ ) and incubated for $12 \mathrm{~h}$ at $37^{\circ} \mathrm{C}$.

Table 1. Strains and plasmids used in this study

\begin{tabular}{|c|c|c|}
\hline Strains and plasmids & Properties & Source \\
\hline \multicolumn{3}{|l|}{ Strain } \\
\hline E. coli DH5a & 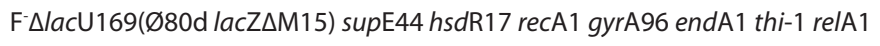 & Invitrogen ${ }^{* *}$ \\
\hline B. subtilis $1 \mathrm{~A} 751$ & eglS $\Delta 102 \mathrm{bglT} / \mathrm{bglS} \Delta$ EVaprEnprE his & $\mathrm{BGSC}^{* * *}$ \\
\hline \multicolumn{3}{|l|}{ Plasmid } \\
\hline pMA5 & E. coli/B. subtilis shuttle vector, $\mathrm{P}_{H p a \Pi^{\prime}} ; \mathrm{Ap}^{\mathrm{r}}, \mathrm{Km}^{\mathrm{r}}$ & Lab stock \\
\hline pMA5-AmyS & pMA5 derivative, amyS from B. stearothermophilus & Lab stock \\
\hline pMA5-AmyS $\Delta$ R179-G180 & pMA5-AmyS* derivative, AmyS $\Delta$ R179-G180 & This work \\
\hline
\end{tabular}

*AmyS GenBank: AF032864.1, ${ }^{* *}$ Invitrogen, Carlsbad, CA, USA, ***BGSC=Bacillus Genetic Stock Center, Columbus, OH, USA 
Expression and purification of AmyS and AmyS mutant, and electrophoresis

The plasmids pMA5-AmyS and pMA5-AmySAR179-G180 were individually transformed into $B$. subtilis $1 A 751$ for protein expression. B. subtilis $1 \mathrm{~A} 751$ cells harbouring pMA5-AmyS and pMA5-AmyS $\triangle R 179-G 180$ were grown overnight and 1-mL culture was transferred into $100 \mathrm{~mL}$ of SR medium containing kanamycin $(25 \mu \mathrm{g} / \mathrm{mL})$ and the culture was then incubated at $37^{\circ} \mathrm{C}$ for $36 \mathrm{~h}$. The supernatant was collected by centrifugation $\left(4^{\circ} \mathrm{C}, 5000 \times \mathrm{g}, 10 \mathrm{~min}\right)$ using centrifuge model $5810 \mathrm{R}$ (Eppendorf, Hamburg, Germany). The enzyme in the supernatant was precipitated by adding solid ammonium sulfate to $70 \%$ (by mass per volume). This was performed at $4{ }^{\circ} \mathrm{C}$ with continuous stirring and then kept on ice for $30 \mathrm{~min}$. The precipitated proteins were collected by centrifugation $\left(4{ }^{\circ} \mathrm{C}, 12000 \times g\right.$, $30 \mathrm{~min}$; model 5810R; Eppendorf), dialyzed against $100 \mathrm{mM}$ sodium acetate $(\mathrm{pH}=6)$, and then concentrated to minimum volume. The purified enzymes were analyzed by SDS-PAGE using the NuPAGE $12 \%$ Bis-Tris Gel together with MOPS SDS Running Buffer. Protein bands were visualized by Coomassie Brilliant Blue R-250 staining.

\section{a-Amylase activity assay}

a-Amylase activity was measured according to the 3,5-dinitrosalicylic acid (DNS) method (14). One unit of a-amylase activity was defined as the amount of enzyme that liberates $1 \mu \mathrm{moL}$ of glucose per min under the detection conditions. In our experiment, $40 \mu \mathrm{L}$ of diluted enzyme solution were mixed with $80 \mu \mathrm{L}$ of $100 \mathrm{mM}$ sodium acetate $(\mathrm{pH}=6)$ and $80 \mu \mathrm{L}$ of $1 \%$ (by mass per volume) soluble starch and then incubated for $20 \mathrm{~min}$ at $75^{\circ} \mathrm{C}$. The reaction was stopped by quenching on ice, after which $200 \mu \mathrm{L}$ of $1 \%$ DNS were added and the sample was boiled for $10 \mathrm{~min}$. Absorbance was measured at $540 \mathrm{~nm}$ using Microplate Reader SpectraMax M5 (Molecular Devices LLC, San Jose, CA, USA) after cooling to room temperature. A reaction mixture without the enzyme was prepared as a negative control. All measurements were performed in triplicate.

\section{Assay for optimal temperature and $\mathrm{pH}$}

The a-amylase activity at 50, 55, 60, 65, 70, 75, 80, 85 and $90^{\circ} \mathrm{C}$ was determined, and the optimal temperature was obtained. a-Amylase activity was measured at $\mathrm{pH}=4.0,4.5,5.0$, $5.5,6.0,6.5,7.0$ and 7.5 and the optimal $\mathrm{pH}$ was determined. The buffers used in the experiment were as follows: $100 \mathrm{mM}$ sodium acetate buffer ( $\mathrm{pH}=4.0$ to 6.0 ) and $100 \mathrm{mM}$ sodium phosphate buffer ( $\mathrm{pH}=6.0$ to 7.5 ).

\section{Thermostability evaluation}

The purified enzyme was incubated with different concentrations of $\mathrm{CaCl}_{2}(0,0.25,0.5$ and $1 \mathrm{mmol} / \mathrm{L})$ at $100{ }^{\circ} \mathrm{C}$ for $0-1 \mathrm{~h}$. Subsequently, the residual enzyme activity was assayed to analyze thermostability.

\section{Computer modelling methods}

The homology models of native AmyS and mutant AmyS$\Delta R 179-G 180$ were created with Discovery Studio v. 4.1 (15), using the wild type BStA (1HVX PDB entry) as a template. The structure was viewed using the PyMol editor (16).

\section{Fed-batch cultivation}

B. subtilis 1 A751 strain harbouring pMA5-AmyS$\triangle R 179-G 180$ was used for high density fermentation in a 7.5-litre BIO FLO 310 fermentor (New Brunswick Scientific Co Inc., Edison, NJ, USA), with a fed-batch strategy. The medium was supplemented with $2.5 \%$ yeast extract, $1.5 \%$ peptone, $0.3 \%$ $\mathrm{K}_{2} \mathrm{HPO}_{4}$ and $50 \mu \mathrm{g} / \mathrm{mL}$ of kanamycin. The airflow rate was controlled at $6.0 \mathrm{~L} / \mathrm{min}$ and, by automatic regulation of stirring speed, dissolved oxygen tension was kept between 20 and 40 $\%$ air saturation. Temperature and $\mathrm{pH}$ were controlled at $37^{\circ} \mathrm{C}$ and 7.0, respectively. A silicone-based anti-foaming agent was used to eliminate foam. Fermentation was carried out with a working volume of $3.5 \mathrm{~L}$ at $37^{\circ} \mathrm{C}$ for $72 \mathrm{~h}$. When cell growth rate reached a constant level, fed-batch cultivation was initiated. Soluble starch ( $8.0 \%$ by mass per volume) was added as the substrate at a constant flow rate until its final mass per volume ratio reached $4.0 \%$. Cell growth was monitored by measuring cell dry mass. a-Amylase activity was analyzed by measuring the cell-free supernatant.

\section{Statistical analysis}

The experiments (a-amylase activity assay and dry cell mass measurement) were performed in triplicates. Data are presented as mean relative value \pm standard deviation.

\section{RESULTS AND DISCUSSION}

\section{Expression of AmyS and AmyS $\triangle R 179-G 180$ in B. subtilis}

In our previous study, we cloned the a-amylase gene (GenBank: AAB86961.1; 17) from a wild type B. stearothermophilus strain and successfully expressed this a-amylase (AmyS) in $B$. subtilis (18). According to sequence alignment, we found that an Arg-Gly peptide present in AmyS was similar to that in BAA (7) and LAMY (8). The deletion of the Arg-Gly peptide greatly improved the thermostability of BAA and LAMY. Therefore, we attempted to optimize AmyS by deleting amino acid residues Arg 179 and Gly 180 simultaneously by site-directed mutagenesis. The mutation ( $\triangle \mathrm{R} 179-\mathrm{G} 180)$ was introduced into AmyS using the Easy Mutagenesis System (TransGen Biotech) and the altered sequence was confirmed by sequencing. The resultant plasmid pMA5-AmyS $\triangle R 179-G 180$ was transformed into B. subtilis $1 \mathrm{~A} 751$ cells. The transformants were cultivated in $30 \mathrm{~mL}$ of SR medium in $250-\mathrm{mL}$ shake flasks at $37^{\circ} \mathrm{C}$ for $36 \mathrm{~h}$. As shown in Fig. 1a, a protein band of approx. $58 \mathrm{kDa}$ was present, which was consistent with the expected size of AmyS. In addition, the proteins of interest, AmyS and AmyS$\triangle R 179-G 180$, were purified to near homogeneity by saturated ammonium sulphate precipitation (Fig. 1b). 

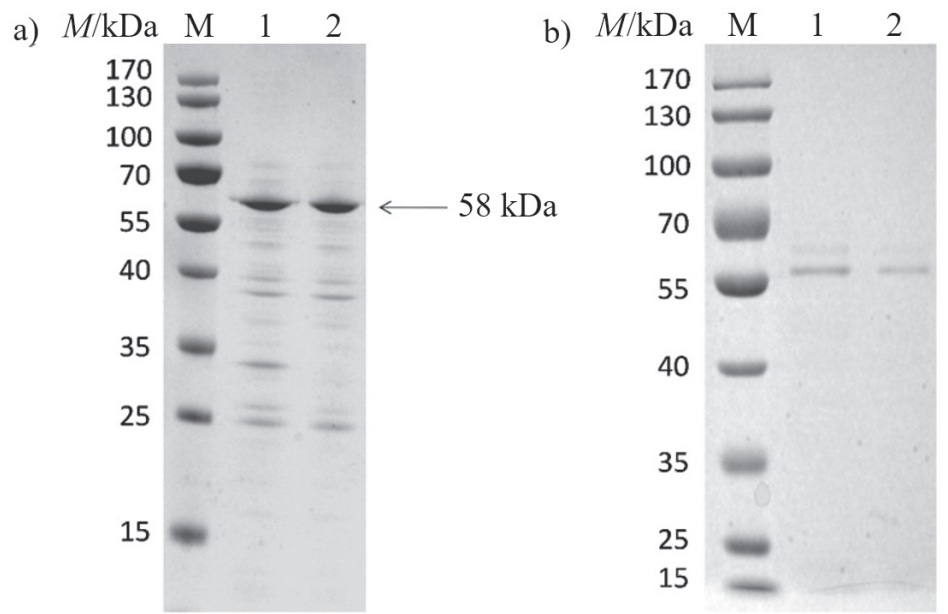

Fig. 1. SDS-PAGE analysis: a) secretion of AmyS and AmyS $\triangle R 179-G 180$ in Bacillus subtilis 1A751, and b) purified AmyS and AmyS $\triangle R 179-G 180$. Lane $\mathrm{M}=$ molecular mass marker, lane 1=AmyS, lane 2=AmyS $\triangle \mathrm{R} 179-\mathrm{G} 180$

\section{Effects of temperature and $\mathrm{pH}$ on AmyS and AmyS mutant properties}

After purification of the enzymes, the enzymatic properties of AmyS and AmySAR179-G180 were evaluated. The optimal temperature and $\mathrm{pH}$ for AmyS and AmyS $\Delta \mathrm{R} 179-\mathrm{G} 180$ activity were assayed. As shown in Fig. 2a, wild type AmyS maintained biological activity within a temperature range of 50 to $90^{\circ} \mathrm{C}(\mathrm{pH}=6.0)$ and showed the highest a-amylase activity at $75^{\circ} \mathrm{C}$. The mutant AmyS $\triangle \mathrm{R} 179-\mathrm{G} 180$ exhibited a similar temperature-activity curve to the wild type AmyS $(\mathrm{pH}=6.0)$, indicating that the mutation in AmyS had no significant effect on reaction temperature. In addition, the effect of $\mathrm{pH}$ on the wild type and mutant AmyS was measured (Fig. 2b). The wild type AmyS was active within a pH range of 4.0 to 7.5 (at $70{ }^{\circ} \mathrm{C}$ ) and the optimal $\mathrm{pH}$ of AmyS was 6.0 . However, it is worth noting that the mutant exhibited its highest activity at $\mathrm{pH}=5.5$, suggesting that the optimal $\mathrm{pH}$ decreased by deleting R179-G180 in AmyS. Moreover, the mutant seemed to be more acid-resistant at $\mathrm{pH}=4.5-6.0$. Considering that $\mathrm{a}$-amylases ideally function at $\mathrm{pH}=4.5$ in industry for the liquefaction of starch, the mutant AmyS $\triangle$ R179-G180 was, therefore, more suitable for future industrial applications.

\section{Enhancement of AmyS thermostability by deleting residues R179-G180}

In order to study the effect of deleting residues R179-G180 on AmyS thermostability, the half-lives of AmyS and AmyS$\triangle \mathrm{R} 179-\mathrm{G} 180$ were determined at high temperatures. As shown in Fig. 3, AmyS showed a time-dependent decrease in amylolytic activity and the half-life was $24 \mathrm{~min}$ at $100^{\circ} \mathrm{C}$. However, AmyS mutant was found to exhibit increased thermostability and the half-life reached $33 \mathrm{~min}$ at the same temperature. In order to investigate the cause of the increased thermostability, we created a homology model of AmyS using Discovery Studio v. 4.1 (15) and compared the model with BLA (Fig. 4). We noticed a small extra loop containing Arg179-Gly180 located in domain B of AmyS. It has been reported
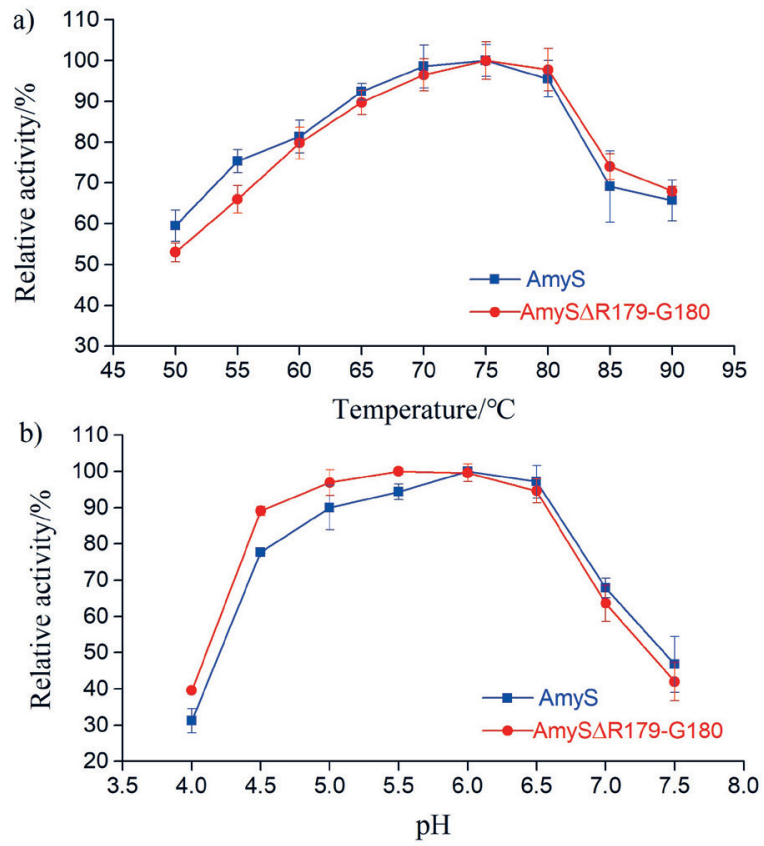

Fig. 2. Effects of: a) temperature, and b) pH on the activity of the wild type and mutant AmyS

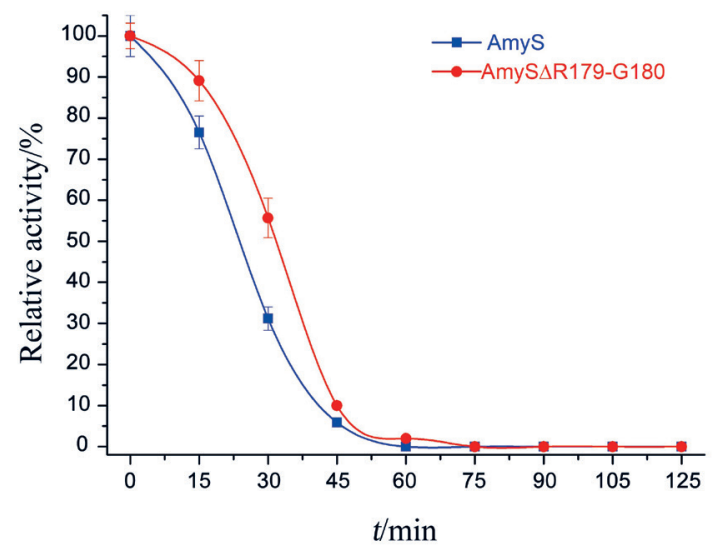

Fig. 3. Thermostability of AmyS and AmyS $\triangle R 179-G 180$. The residual activity is shown as the relative activities after incubation at $100^{\circ} \mathrm{C}$ for different times 
that domain B is likely to be responsible for the differences in substrate specificity and thermostability between different a-amylases. In addition, Suzuki et al. (7) demonstrated that the thermostability of BAA was greatly improved by deletion of Arg176-Gly177, in a similar loop, along with the replacement of Ala269 with Lys269 using site-directed mutagenesis. According to sequence alignment and protein structure comparison, other a-amylases, including LAMY from Bacillus KSM-1378 (8), BStA from B. stearothermophilus US100 (10) and BAC $\triangle N C$ from Bacillus sp. strain TS-23 (9), also resulted in similar improvements in enzyme properties by the deletion of amino acids in the same region.

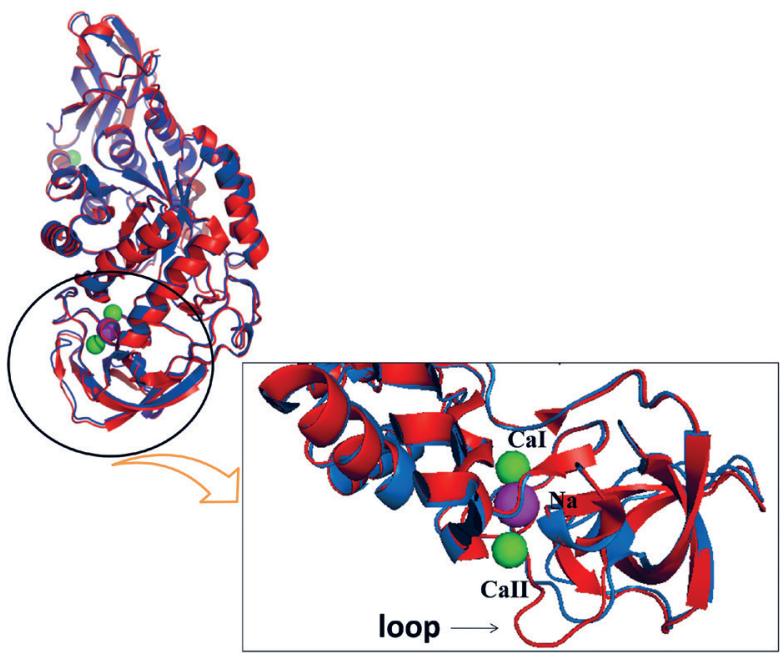

Fig. 4. Loop region with superimposition of AmyS and BLA, focusing on the loop region (Arg179-Gly180 using AmyS numbering). BLA (blue), AmyS (red), sodium ion (magenta sphere), and calcium ions (green spheres)

\section{Decrease in AmyS $\triangle R 179-G 180$ calcium requirements}

We compared the structure of the AmyS model with that of the AmyS $\triangle \mathrm{R} 179-\mathrm{G} 180$ model and found that the deletion of R179-G180 caused a slight structural rearrangement. Ali et al. (10) confirmed that the Call site in the AmyUS100AI214-G215 model lost interactions with Asp105 and His238, which contributes to the maintenance of connections between the $A$ and $B$ domains. They also found that the deletion of I214-G215 in AmyUS100 not only increased enzyme thermostability, but also significantly decreased calcium requirements. It was thus possible that the deletion of R179-G180 affects the calcium-binding sites in AmyS. Therefore, calcium requirements of AmyS and AmyS $\triangle R 179-G 180$ were tested through comparison of enzymatic activity of residue following incubation of the enzyme with different concentrations of calcium at 100 ${ }^{\circ} \mathrm{C}$. As shown in Fig. 5, the maximal thermostability of AmyS (Fig. 5a) was obtained with $1 \mathrm{mmol} / \mathrm{L}$ of $\mathrm{CaCl}_{2}$, however, the maximal thermostability of AmyS $\triangle \mathrm{R} 179-\mathrm{G} 180$ (Fig. 5b) was obtained with only $0.25 \mathrm{mmol} / \mathrm{L}$ of $\mathrm{CaCl}_{2}$. This observation confirmed the above hypothesis. a)

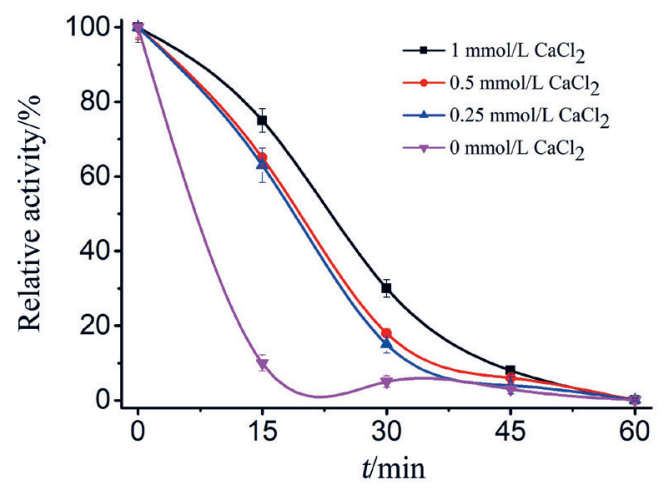

b)

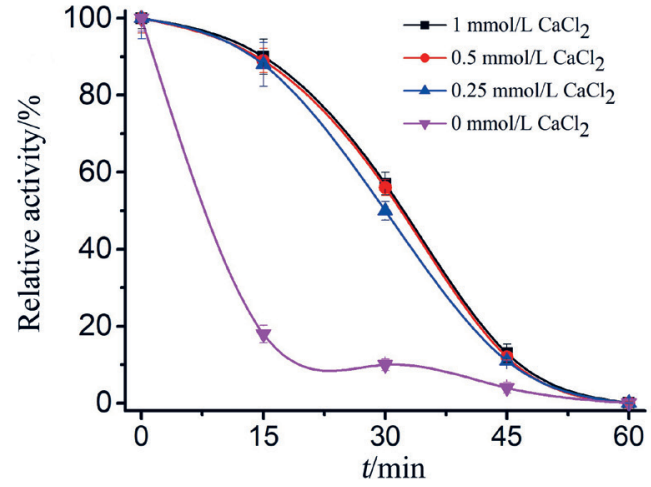

Fig. 5. Effect of R179-G180 deletion on calcium demand. Comparison of the thermostability of: a) AmyS and b) AmyS $\triangle$ R179-G180 at $100{ }^{\circ} \mathrm{C}$ and $\mathrm{pH}=5.5$ with different $\mathrm{CaCl}_{2}$ concentrations

It is well-known that a-amylases usually contain calcium-binding sites to stabilize the interface between the highly homologous domain $\mathrm{A}$ and the more variable domain $\mathrm{B}$ (19). Declerck et al. (20) reported that the interface was sensitive to structural disruption and confirmed that when BLA was exposed to high temperature, protein unfolding began in this region. Therefore, they speculated that any mutation that altered or reinforced stabilizing interactions in this region may influence the rate of thermal inactivation (21). There are several reasons to illustrate increased thermostability. Suzuki et al. (7) confirmed that increased thermostability of BAA by deletion of Arg176-Gly177 could be attributed to an increase in hydrophobicity and the charged residues changing into non-polar ones. Igarashi et al. (8) reported that the thermostability of LAMY improved with enhanced calcium binding of the enzyme. In the present study, the increased thermostability of AmyS $\Delta$ R179-G180 may be caused by changing the calcium-binding sites.

\section{Production of AmySAR179-G180 with fed-batch cultivation in a 7.5-litre fermentor}

Since the AmyS $\Delta$ R179-G180 exhibited better enzymatic properties and was, thus, able to be applied in the industrial production of sugars, the expression efficiency of $B$. subtilis 1 A751 containing the plasmid pMA5-AmyS $\triangle \mathrm{R} 179-\mathrm{G} 180$ was evaluated for high density fermentation. A volume of 1 $\mathrm{mL}$ of frozen glycerol stock was inoculated into $100 \mathrm{~mL}$ of SR 
medium and incubated for $18 \mathrm{~h}$ at $37^{\circ} \mathrm{C}$. Next, $5 \%$ (by volume) of seed culture was inoculated into a 7.5 -litre fermentor supplemented with fermentation medium. When the rate of cell growth became constant, fed-batch cultivation was initiated. Soluble starch ( $8 \%$ by mass per volume) was added as the substrate at a constant flow rate until its final mass per volume ratio reached $4.0 \%$. For AmyS $\Delta$ R179-G180 production (Fig. 6), the maximum biomass concentration reached $32.3 \mathrm{~g} / \mathrm{L}$ after $36 \mathrm{~h}$. The maximum enzyme activity reached $3300 \mathrm{U} / \mathrm{mL}$ after $72 \mathrm{~h}$ and the productivity level reached $45.8 \mathrm{U} /(\mathrm{mL} \cdot \mathrm{h})$.

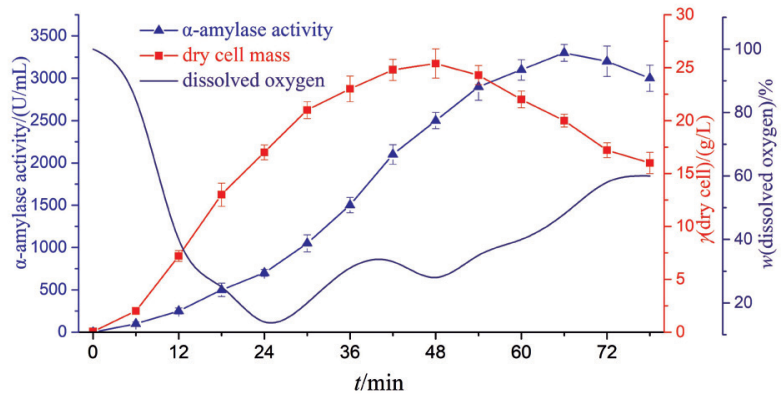

Fig. 6. Production of a-amylase in the recombinant strain $1 A 751$ harbouring pMA5-AmyS $\triangle$ R179-G180 using a fed-batch strategy in a 7.5-litre fermentor

\section{CONCLUSIONS}

By deleting the amino acid residues arginine 179 and glycine 180, the thermostability of the a-amylase AmyS from Bacillus stearothermophilus was enhanced and the half-life at $100^{\circ} \mathrm{C}$ was significantly increased. AmyS $\Delta \mathrm{R} 179-\mathrm{G} 180$ acquired greater acid resistance and lower calcium requirements than the wild type AmyS to maintain a-amylase activity. Finally, we evaluated the secretory capacity of the recombinant strain in a 7.5-litre fermentor by fed-batch fermentation and observed a high level of a-amylase activity.

\section{ACKNOWLEDGEMENTS}

This work was supported by Natural Science Foundation of Tianjin City, PR China (16JCYBJC23500), Tianjin Science Fund for Distinguished Young Scholars (17JCJQJC45300), Tianjin Science and Technology Project (15PTCYSY00020), the Key Projects in the Tianjin Science and Technology Pillar Program (14ZCZDSY00058) and Key Laboratory of Systems Microbial Biotechnology, Tianjin Institute of Industrial Biotechnology, Chinese Academy of Sciences.

\section{REFERENCES}

1. Pandey A, Nigam P, Soccol CR, Soccol VT, Singh D, Mohan R. Advances in microbial amylases. Biotechnol Appl Biochem. 2000;31:135-52.

https://doi.org/10.1042/BA19990073

2. Richardson TH, Tan X, Frey G, Callen W, Cabell M, Lam D, et al. A novel, high performance enzyme for starch liquefaction - discovery and optimization of a low $\mathrm{pH}$, thermostable a-amylase. J Biol Chem. 2002;277:26501-7.

https://doi.org/10.1074/jbc.M203183200

3. Alikhajeh J, Khajeh K, Ranjbar B, Naderi-Manesh H, Lin $\mathrm{YH}$, Liu EH, et al. Structure of Bacillus amyloliquefaciens a-amylase at high resolution: Implications for thermal stability. Acta Cryst. 2010;66:121-9. https://doi.org/10.1107/S1744309109051938

4. Suvd D, Fujimoto Z, Takase K, Matsumura M, Mizuno H. Crystal structure of Bacillus stearothermophilus a-amylase: Possible factors determining the thermostability. J Biochem. 2001;129:461-8.

https://doi.org/10.1093/oxfordjournals.jbchem.a002878

5. Saito N. A thermophilic extracellular a-amylase from Bacillus licheniformis. Arch Biochem Biophys. 1973;155:290-8. https://doi.org/10.1016/0003-9861(73)90117-3

6. Cherry JR, Fidantsef AL. Directed evolution of industrial enzymes: An update. Curr Opin Biotechnol. 2003;14:438-43. https://doi.org/10.1016/S0958-1669(03)00099-5

7. Suzuki Y, Ito N, Yuuki T, Yamagata H, Udaka S. Amino acid residues stabilizing a Bacillus alpha-amylase against irreversible thermoinactivation. J Biol Chem.1989;264:18933-8.

8. Igarashi K, Hatada Y, Ikawa K, Araki H, Ozawa T, Kobayashi $\mathrm{T}$, et al. Improved thermostability of a Bacillus a-amylase by deletion of an arginine-glycine residue is caused by enhanced calcium binding. Biochem Biophys Res Commun. 1998;248:372-7.

https://doi.org/10.1006/bbrc.1998.8970

9. Lin LL, Huang CC, Lo HF. Impact of Arg210-Ser211 deletion on thermostability of a truncated Bacillus sp. strain TS-23 a-amylase. Process Biochem. 2008;43:559-65. https://doi.org/10.1016/j.procbio.2008.01.020

10. Ben Ali M, Khemakhem B, Robert X, Haser R, Bejar S. Thermostability enhancement and change in starch hydrolysis profile of the maltohexaose-forming amylase of Bacillus stearothermophilus US100 strain. Biochem J. 2006;394:51-6. https://doi.org/10.1042/BJ20050726

11. Green MR, Sambrook J. Molecular cloning: A laboratory manual. New York, USA: Cold Spring Harbor Laboratory Press; 2012.

12. Sanger F, Nicklen $S$, Coulson AR. DNA sequencing with chain-terminating inhibitors. Proc Natl Acad Sci USA. 1977;74:5463-7.

13. Yasbin RE, Wilson GA, Young FE. Transformation and transfection in lysogenic strains of Bacillus subtilis: Evidence for selective induction of prophage in competent cells. J Bacteriol. 1975;121:296-304.

14. Rashid N, Ahmed N, Saleem Haider M, Haque I. Effective solubilization and single-step purification of Bacillus licheniformis a-amylase from insoluble aggregates. Folia Microbiol. 2010;55:133-6. https://doi.org/10.1007/s12223-010-0020-y

15. Discovery Studio, v. 4.1, San Diego, CA, USA: 2014. Available from: http://accelrys.com. 
16. PyMOL Molecular Graphics System, v. 1.8.6, Schrödinger, LLC, Cambridge, MA, USA; 2017. Available from: https:// www.pymol.org.

17. Da Silva ACR, Fernandes E, Pueyo MT. Alpha amylase [Geobacillus stearothermophilus]. GenBank: AAB86961.1. Bethesda, MD, USA: National Center for Biotechnology Information; 1997. Available from: https://www.ncbi.nlm.nih. gov/protein/2642326.

18. Chen J, Fu G, Gai Y, Zheng P, Zhang D, Wen J. Combinatorial Sec pathway analysis for improved heterologous protein secretion in Bacillus subtilis: Identification of bottlenecks by systematic gene overexpression. Microb Cell Fact. 2015;14:92.

https://doi.org/10.1186/s12934-015-0282-9
19. Machius M, Wiegand G, Huber R. Crystal structure of calcium-depleted Bacillus licheniformis a-amylase at 2.2 Å resolution. J Mol Biol. 1995;246:545-59.

https://doi.org/10.1006/jmbi.1994.0106

20. Declerck N, Machius M, Wiegand G, Huber R, Gaillardin C. Probing structural determinants specifying high thermostability in Bacillus licheniformis a-amylase. J Mol Biol. 2000;301:1041-57.

https://doi.org/10.1006/jmbi.2000.4025

21. Declerck N, Machius M, Joyet P, Wiegand G, Huber R, Gaillardin C. Engineering the thermostability of Bacillus licheniformis a-amylase. Biologia. 2002;57:203-11. 